\title{
Stability of quantum states of finite macroscopic systems 6
}

\author{
Akira Shimizu, Takayuki Miyadera and Akihisa Ukena \\ Department of Basic Science, University of Tokyo \\ 3-8-1 Komaba, Tokyo 153-8902, Japan \\ E-mail:shmz@ASone.c.u-tokyo.ac.jp
}

\begin{abstract}
We study the stabilities of quantum states of macroscopic systems, against noises, against perturbations from environments, and against local measurements. We show that the stabilities are closely related to the cluster property, which describes the strength of spatial correlations of fluctuations of local observables, and to fluctuations of additive operators. The present theory has many applications, among which we discuss the mechanism of phase transitions in finite systems and quantum computers with a huge number of qubits.
\end{abstract}

\section{INTRODUCTION}

The stability of quantum states of macroscopic systems, which are subject to noises or perturbations from environments, have been studied in many fields of physics, including studies of 'macroscopic quantum coherence' [1] and quantum measurement. [2] However, most previous works assumed that the principal systems were describable by a small number of collective coordinates. Although such models might be applicable to systems which have a nonnegligible energy gap to excite 'internal coordinates' of the collective coordinates, there are many systems that do not have such an energy gap. As a result of the use of such simple models, the results depended strongly on the choices of the coordinates and the form of the interaction $\hat{H}_{\text {int }}$ between the principal system and a noise or an environment. For example, a robust state for some $\hat{H}_{\text {int }}$ can become a fragile state for another $\hat{H}_{\text {int }}$. However, macroscopic physics and experiences indicate that a more universal result should be drawn for the stability of quantum states of macroscopic systems. Moreover, the stability against measurements were not studied well. Although one might conjecture that effects of measurements would be equivalent to effects of noises or environments, the conjecture is wrong as we will show in section 9 of this paper.

In this paper, we study these stabilities using a general model with a macroscopic number of degrees of freedom $N$. In addition to the fact that $N$ is huge, we make full use of the locality - 'additive' observables must be the sum of local observables over a macroscopic region (Eq. (7) below), the interaction $\hat{H}_{\text {int }}$ must be local (Eqs. (12) and (144)), and measurement must be local. By noticing these points, which were absent or ambiguous in most previous works, we derive general and universal results. [3] We also propose a new criterion of stability; the stability against local measurements. We present a general and universal result also for this stability. [3]

The present theory has many applications because it is general and universal. We here mention applications to phase transitions in finite systems, and quantum computers with a huge number of qubits.

\section{MACROSCOPIC QUANTUM SYSTEMS}

We consider "macroscopic quantum systems." We first describe what this means.

As usual, we are only interested in phenomena in some energy range $\Delta E$, and describe the system by an effective theory which correctly describes the system only in $\Delta E$. For a given $\Delta E$, let $\mathcal{M}$ be the number of many-body quantum states in that energy range. Then,

$$
N \sim \ln \mathcal{M}
$$

is the degrees of freedom of the effective theory. Here, the symbol ' $\sim$ ' means that corrections of $o(N)$, such as $\ln (\Delta E)=O(\ln N)$, are neglected. For weakly interacting systems, for example, $N$ becomes the number of single-body quantum states which constitutes the $\mathcal{M}$ many-body states. Note that $N$ sometimes becomes a small number even for a system of many degrees of freedom when, e.g., a non-negligible energy gap exists in $\Delta E$. Such systems include some SQUID systems at low temperatures, and a heavy atom at a meV or lower energy range. We here exclude

\footnotetext{
*Proceedings of the Japan-Italy Joint Waseda Workshop on Fundamental Problems in Quantum Mechanics, 27-29 September, 2001, Tokyo, Japan. (Edited by S. Tasaki, To be published from World Scientific, 2002)
} 
such systems, because they are essentially systems of small degrees of freedom. Namely, we consider systems whose $N$ is a macroscopic number. Otherwise, the difference between $O(N)$ and $O\left(N^{2}\right)$, which plays the central roles in macroscopic physics and in the following discussions, would be irrelevant. Since $\Delta E$ sets a minimum length scale $\ell$, the system extends spatially over a finite volume

$$
V \sim N \ell^{d}
$$

in $d$ dimension. Since $V$ is proportional to the macroscopic number $N$, we say $V$ is also macroscopic, disregarding the magnitude of $\ell$.

In short, we consider macroscopic quantum systems for which $N$ and $V$ are macroscopic for a given energy range $\Delta E$. Note that $V$ is essentially equal to $N$ because of Eq. (2). We therefore use $V$ and $N$ (and the words "volume" and "degrees of freedom") interchangeably in the following discussions.

\section{CLUSTER PROPERTY}

As we shall show later, correlations between distant points are important in the study of stability. As a measure of the correlations, we first consider the cluster property. Although we are considering finite systems, we first review the case of infinite systems. [- A quantum state of an in infinite system is said to have the cluster property if

$$
\langle\delta \hat{a}(x) \delta \hat{b}(y)\rangle \rightarrow 0 \text { as }|x-y| \rightarrow \infty
$$

for any local operators $\hat{a}(x)$ and $\hat{b}(y)$ at $x$ and $y$, respectively, where

$$
\begin{aligned}
\delta \hat{a}(x) & \equiv \hat{a}(x)-\langle\hat{a}(x)\rangle, \\
\delta \hat{b}(y) & \equiv \hat{b}(y)-\langle\hat{b}(y)\rangle .
\end{aligned}
$$

Here, by a local operator at $x$ we mean a finite-order polynomial of field operators and their finite-order derivatives at position $x .1$ The cluster property should not be confused with the lack of long-range order: A state with a long-range order can have the cluster property. In fact, symmetry-breaking vacua of infinite systems have both the long-range

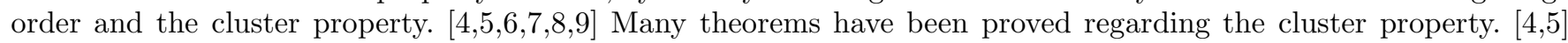
Among them, the following is most important to the present theory:

Known theorem: In infinite systems, any pure state has the cluster property.

In oder to study finite systems, we generalize the concept of the cluster property to the case of finite systems. For a small positive number $\epsilon$, let $\Omega(\epsilon)$ be the minimum size of the region over which correlations of any local operators become smaller than $\epsilon$. Namely, $\Omega(\epsilon) \equiv \sup _{x}|\Omega(\epsilon, x)|$, where $|\Omega(\epsilon, x)|$ denotes the size of the region $\Omega(\epsilon, x)$. Here, $\Omega(\epsilon, x)$ is defined by its complement $\Omega(\epsilon, x)^{c}$, which is the region of $y$ in which

$$
|\langle\delta \hat{a}(x) \delta \hat{b}(y)\rangle| \leq \epsilon \sqrt{\left\langle\delta \hat{a}^{\dagger}(x) \delta \hat{a}(x)\right\rangle\left\langle\delta \hat{b}^{\dagger}(y) \delta \hat{b}(y)\right\rangle}
$$

for any local operators $\hat{a}(x)$ and $\hat{b}(y)$. Then, we say that a quantum state of a finite macroscopic system has the cluster property if $\Omega(\epsilon) \ll V$ for sufficiently large $V$. More strictly, we consider a sequence of states for various values of $V$ such that they are essentially equivalent to each other except for the values of $V$ 目 We say that the states (for large $V$ ) of the sequence have the cluster property if $\Omega(\epsilon)$ for any $\epsilon>0$ becomes independent of $V$ for sufficiently large $V$. It is clear from the known theorem above that a sequence of pure states of finite $V$ that do not have the cluster property approaches a mixed state of an infinite system as $V \rightarrow \infty$.

\section{ANOMALOUSLY-FLUCTUATING STATES}

As a second measure of correlations between distant points, we consider fluctuations of additive operators. Here, by an additive operator we mean an operator of the following form:

\footnotetext{
${ }^{1}$ To express and utilize the locality of the theory manifestly, we use a field theory throughout this paper.

${ }^{2}$ For example, the ground-state wavefunctions of many particles for the same particle density, for various values of $V$.
} 


$$
\hat{A}=\sum_{x \in V} \hat{a}(x)
$$

where $\hat{a}(x)$ denotes a local operator at $x$. When we regard the system as a composite system of subsystems 1 and 2 , then $\hat{A}=\hat{A}^{(1)}+\hat{A}^{(2)}$, hence the name "additive." In thermodynamics, it is assumed that

$$
\left\langle\delta A^{2}\right\rangle \leq o\left(V^{2}\right)
$$

for all additive quantities. 3 In particular, if a state (classical or quantal) satisfies

$$
\left\langle\delta A^{2}\right\rangle \leq O(V)
$$

for all additive quantities, we call it a normally-fluctuating state (NFS). In quantum theory of finite macroscopic systems, however, there exist pure states for which some of additive operators have anomalously-large fluctuations;

$$
\left\langle\delta \hat{A}^{2}\right\rangle=O\left(V^{2}\right)
$$

We call such a pure state an anomalously-fluctuating state (AFS). 目

It is easy to show that an AFS does not have the cluster property. Hence, according to the known theorem of section 3, an AFS cannot be a pure state in infinite systems. Considering also that in thermodynamics any state in a pure phase is an NFS, we see that an AFS can exist only in finite macroscopic quantum systems. Since AFSs are such unusual states, they might be expected to be unstable in some sense. Our purpose is to study this conjecture, by formulating the stability definitely, and thereby present general theorems about the stability.

\section{FRAGILITY OF QUANTUM STATES OF MACROSCOPIC SYSTEMS}

We say a quantum state is fragile if its decoherence rate $\Gamma$ satisfies

$$
\Gamma \sim K V^{1+\delta}
$$

where $K$ is a function of microscopic parameters, and $\delta$ is a positive constant.

To understand the meaning of the fragility, consider first the non-fragile case where $\delta=0$. In this case, the decoherence rate per unit volume is independent of $V$. This is a normal situation in the sense that the total decoherence rate $\Gamma$ is basically the sum of local decoherence rates, which are determined only by microscopic parameters (i.e., independent of $V$ ). On the other hand, the case $\delta>0$ is an anomalous situation in which the decoherence rate per unit volume behaves as $\sim K V^{\delta}$. Note that this can be very large even when $K$ is small, because $V$ is huge. This means that a fragile quantum state of a macroscopic system decoheres due to a noise or environment at an anomalously great rate, even when the coupling constant between the system and the noise or environment is small.

\section{FRAGILITY IN WEAK NOISES}

The most important assumption of the present theory is the locality. For the interaction with a noise, the locality requires that the interaction Hamiltonian should be the sum of local interactions;

$$
\hat{H}_{\mathrm{int}}=\sum_{x \in V} f(x, t) \hat{a}(x) .
$$

Here, $f(x, t)$ is a random noise field with vanishing average $\overline{f(x, t)}=0$, and $\hat{a}(x)$ is a local operator at $x$. (See section 3 for the meaning of the local operator.) We assume that the statistics of $f(x, t)$ is translationally invariant both

\footnotetext{
${ }^{3}$ Otherwise, it would be meaningless to talk about the average value of $A$, which is $O(V)$.

${ }^{4}$ It is possible to change an AFS into an NFS by enlarging the system by adding an extra system of volume $V_{\text {extra }} \sim V^{2}$, in which the quantum state is an NFS, because this leads to $\left\langle\delta A^{2}\right\rangle=O\left(V^{2}\right)+O\left(V_{\text {extra }}\right)=O\left(V_{\text {extra }}\right) \simeq O\left(V_{\text {extra }}+V\right)$. We here exclude such an artificial and uninteresting case.
} 
spatially and temporally, i.e., $\overline{f(x, t) f\left(x^{\prime}, t^{\prime}\right)}$ is a function of $x-x^{\prime}$ and $t-t^{\prime}$. We also assume that the time correlation of the noise is short.

The total Hamiltonian is

$$
\hat{H}_{\text {total }}=\hat{H}+\hat{H}_{\text {int }} \text {. }
$$

Here, $\hat{H}$ denotes the Hamiltonian of the principal system, which can be a general Hamiltonian including, e.g., manybody interactions. Using this general local model, we can show the following for the fragility that is defined in section 5:

Theorem 1: Let $|\Psi\rangle$ be a pure state, whose time evolution by $\hat{H}$ is slow, of a macroscopic system. If $|\Psi\rangle$ is an AFS, then it is fragile in the presence of some weak noise. If $|\Psi\rangle$ is an NFS, then it is not fragile in any weak noise.

It follows from this theorem that an AFS decoheres (hence collapses) at an anomalously great rate if external noises contain such a noise component, whereas a NFS does not decohere at such an anomalously great rate in any weak noise.

\section{FRAGILITY UNDER WEAK PERTURBATIONS FROM ENVIRONMENTS}

The physical realities of noises are perturbations from environments. We can show a similar theorem for the effects of perturbations from environments. Again, the most important assumption is the locality of the interaction between the principal system and an environment. Namely, the interaction Hamiltonian should be the sum of local interactions;

$$
\hat{H}_{\mathrm{int}}=\sum_{x \in V} \hat{f}(x) \hat{a}(x) .
$$

Here, $\hat{f}(x)$ and $\hat{a}(x)$ are local operators at $x$ of an environment and the principal system, respectively. Similarly to the case of noise, we assume that (in the interaction picture) $\langle\hat{f}(x, t)\rangle_{\mathrm{E}}=0$, and that $\left\langle f(x, t) f\left(x^{\prime}, t^{\prime}\right)\right\rangle_{\mathrm{E}}$ is a function of $x-x^{\prime}$ and $t-t^{\prime}$, where $\langle\cdots\rangle_{\mathrm{E}}$ denotes the expectation value for the state of the environment $\mathrm{E}$. We also assume that the correlation time of $\left\langle f(x, t) f\left(x^{\prime}, t^{\prime}\right)\right\rangle_{\mathrm{E}}$ is short. The total Hamiltonian is

$$
\hat{H}_{\text {total }}=\hat{H}+\hat{H}_{\mathrm{int}}+\hat{H}_{\mathrm{E}}
$$

where $\hat{H}$ and $\hat{H}_{\mathrm{E}}$ denote the Hamiltonians of the principal system and the environment, respectively. Here, $\hat{H}$ can be a general Hamiltonian including, e.g., many-body interactions. Using this general local model, we can show the following for the fragility that is defined in section 5 :

Theorem 2: Let $|\Psi\rangle$ be a pure state, whose time evolution by $\hat{H}$ is slow, of a macroscopic system. If $|\Psi\rangle$ is an AFS, then it is fragile under some weak perturbation from some environment. If $|\Psi\rangle$ is an NFS, then it is not fragile under any weak perturbations from environments.

It follows from this theorem that an AFS decoheres (hence collapses) at an anomalously great rate if perturbations from environments contain such a perturbation term, whereas a NFS does not decohere at such an anomalously great rate under any weak perturbations from environments.

\section{DO RELEVANT PERTURBATIONS ALWAYS EXIST?}

By theorems 1 and 2, we have shown that NFSs are not fragile in any noises or environments, which interact weakly with the principal system via any local interactions. This should be contrasted with the results of most previous works, according to which a state could be either fragile or robust depending on the form of the interaction. We have obtained the general and universal conclusion because we have made full use of the locality as well as the huge degrees of freedom.

Regarding AFSs, on the other hand, theorems 1 and 2 show only that they are fragile in some noise or environment, which interact weakly with the principal system via local interactions. In other words, for any AFS it is always possible to construct a noise (or an environment) and a weak local interaction that make the AFS fragile. These theorems do not guarantee the existence of such a relevant noise (or an environment) and a relevant interaction in real physical systems. We discuss this point in this section.

As described in section 2, we are only interested in phenomena in some energy range $\Delta E$, and describe the system by an effective theory which correctly describes the system only in $\Delta E$. The effective theory can be constructed from an elementary dynamics by an appropriate renormalization process. In this process, in general, many interaction 
terms would be generated in the effective interaction $H_{\text {int }} .0$ Hence, it seems quite rare that a relevant noise or an environment and a relevant interaction are completely absent. Even when the coupling constant to the relevant noise (or environment) is, say, ten times smaller than those to other noises (or environments), the relevant noise (or environment) would dominate the decoherence process of the AFS because the decoherence rate grows anomalously fast with increasing $V$, except when the noise (or the perturbation from the environment) is negligibly weak such that its intensity is, e.g., $O(1 / V)$. Namely, an AFS should be fragile apart from such an exceptional case.

However, for general systems, we cannot exclude the exceptional case where the relevant noise is negligibly weak. Therefore, we cannot draw a definite conclusion on whether AFSs are always fragile in real physical systems. This motivates us to explore another stability, which will be described in the next section.

\section{STABILITY AGAINST LOCAL MEASUREMENTS}

We can prove a stronger statement by considering the stability against measurement.

Suppose that one performs an ideal measurement of a local observable $\hat{a}(x)$ at $t=t_{a}$ for a state $\hat{\rho}$ (pure or mixed) of a macroscopic system, and obtains a value $a$. Subsequently, one measures another local observable $\hat{b}(y)$ at a later time $t_{b}$, and obtains a value $b$. Let $P(b ; a)$ be the probability distribution of $b$, i.e., the probability that $b$ is obtained at $t_{b}$ under the condition that $a$ was obtained at $t_{a}$. On the other hand, one can measure $\hat{b}(y)$ at $t=t_{b}$ without performing the measurement of $\hat{a}(x)$ at $t_{a}$. Let $P(b)$ be the probability distribution of $b$ in this case. We say $\hat{\rho}$ is stable against local measurements if for any $\epsilon>0$

$$
|P(b ; a)-P(b)| \leq \epsilon \text { for sufficiently large }|x-y|,
$$

for any local operators $\hat{a}(x)$ and $\hat{b}(y)$ and their eigenvalues $a$ and $b$ such that $P(a) \geq \varepsilon$.

This stability is stronger than the stability against noises and perturbations from environments. In fact, the latter stability is related to $\left|\sum_{a} P(b ; a)-P(b)\right|$. There are many examples of states for which $\left|\sum_{a} P(b ; a)-P(b)\right| \leq \epsilon$ is satisfied whereas $|P(b ; a)-P(b)| \leq \epsilon$ is not.

For the simple case $t_{b}-t_{a} \rightarrow 0$, we can show the following 0

Theorem 3: Let $\hat{\rho}$ be a pure or mixed state of a macroscopic system. If $\hat{\rho}$ is stable against local measurements, then it has the cluster property, and vice versa.

It follows from this theorem that any AFS is unstable against local measurements.

\section{MECHANISM OF SYMMETRY BREAKING IN FINITE SYSTEMS}

AFSs generally appear in, e.g., finite systems which will exhibit symmetry breaking if $V$ goes to infinity. In such systems, we can find states (of finite systems) which approach a symmetry-breaking vacuum as $V \rightarrow \infty$. We call such a state a pure-phase vacuum. It has a finite expectation value $\langle\hat{M}\rangle=O(V)$ of an additive order parameter $\hat{M}$, and has relatively small fluctuations $\left\langle\delta \hat{A}^{2}\right\rangle \leq O(V)$ for any additive operator $\hat{A}$ (including $\hat{M}$ ) [5, 6, 6, 8, 9]. Hence, the pure-phase vacua are NFSs. In a mean-field approximation, the pure-phase vacua have the lowest energy. However, it is always possible to construct a pure state(s) that does not break the symmetry, $\langle\hat{M}\rangle=0$, and has an equal or lower energy than the pure-phase vacua [6,9]. Although such states cannot be pure in infinite systems, they can be pure in finite systems [4.6.9.10]. When $[\hat{H}, \hat{M}] \neq 0$, in particular, the exact lowest-energy state is generally such a symmetric ground state [6.6]:86]. To lower the energy of a pure-phase vacuum, a SB field is necessary. However, an appropriate SB field would not always exist in laboratories. The symmetric ground state is composed primarily of a superposition of pure-phase vacua with different values of $\langle\hat{M}\rangle$, and, consequently, it has an anomalously large fluctuation of $\hat{M}$; $\left\langle\delta \hat{M}^{2}\right\rangle=O\left(V^{2}\right)$. 6, 6, 8,9] Therefore, if one obtains the exact lowest-energy state (e.g., by numerical diagonalization) of a finite system, which will exhibit symmetry breaking if $V$ goes to infinity, the state is often an AFS.

The present results suggest a new origin of symmetry breaking in finite systems. [11] Although symmetry breaking is usually described as a property of infinite systems, it is observed in finite systems as well. The results of sections 6 and 7 suggest that although a pure-phase vacuum (which is an NFS) has a higher energy than the symmetric

\footnotetext{
${ }^{5}$ We expect, in accordance with experiences, that by an appropriate renormalization process $H_{\text {tot }}$ can be made local in the relevant space-time scale.

${ }^{6}$ Results for more general cases will be described elsewhere.
} 
ground state (an AFS), the former would be realized because the latter is fragile in some noises or environments. This mechanism may be called "environment-induced symmetry breaking," a special case of which was discussed for interacting many-bosons. [9, 10,11] The result of section 9 suggests more strongly that only a pure-phase vacuum should be realized, because an AFS is changed into another state when one measures only a tiny part of the system, and such drastic changes continue by repeating measurements, until the state becomes an NFS. This mechanism may be called "measurement-induced symmetry breaking."

We consider that these scenarios explain the symmetry breaking (i.e., realization of an pure-phase vacuum) in finite systems, much more naturally and generally than the idea of the symmetry breaking field: It seems quite artificial to assume that an appropriate static symmetry breaking field would always present in real physical systems, 0 although it is true that symmetry breaking fields are a convenient mathematical tool.

\section{STABILITY OF QUANTUM COMPUTERS WITH MANY QUBITS}

Quantum computers are useful only when the number of qubits $N$ is huge. Hence, useful quantum computers are macroscopic quantum systems.

Various states appear in the course of a quantum computation. Some state may be an NFS, for which $\left\langle\delta \hat{A}^{2}\right\rangle=O(V)$ for any additive operator $A$. This means that correlations between distant qubits are weak. Properties of such states may be possible to emulate by a classical system with local interactions. We therefore conjecture that other states AFSs - should appear in some stages of the computation for a quantum computer to be much faster than classical computers. [13] In fact, two of the authors confirmed this conjecture in Shor's algorithm for factoring. 114]

The present results suggest that the decoherence rate of quantum computers can be estimated by fluctuations of additive operators, which depend strongly on the number of qubits $N$ and the natures of the states of the qubits. 15. Since AFSs are used in some stages of the fast quantum computation, the state of qubits can become fragile in some noise or environment, for quantum computers with many qubits. Note that the dominant perturbation for the case of huge $N$ can be different from that for small $N$, because the decoherence rate of an AFS grows anomalously fast with increasing $N$. Therefore, the quantum computer should be designed in such a way that it utlizes AFSs for which the intensities of the relevant noises are $O(1 / N)$ or smaller. Since the error corrections are not almighty, we think that one must consider both such optimization and the error corrections to realize a quantum computer with a large number of qubits.

\section{DISCUSSIONS}

The present results show that the stabilities of quantum states of finite macroscopic systems are closely related to the cluster property, which describes the strength of spatial correlations of fluctuations of local observables, and to fluctuations of additive operators. Note that the stabilities are defined as dynamical properties of an open system, whereas the cluster property and fluctuations of additive operators are defined as static properties of a closed system. Hence, it is non-trivial - may be surprising - that they are closely related to each other.

We stress that the approximate stability against all local interactions (between the principal system and environments) would be more important than the exact stability against a particular interaction, which was frequently discussed in previous works. As discussed in section 8, many types of interactions would coexist in real physical systems, and the exact stability against one of them could not exclude fragility to another.

In this paper, we did not mention temperature. It is clear that similar conclusions can be drawn for thermal equilibrium states (Kubo-Martin-Schwinger states [4.5), because thermal equilibrium states can be represented as vector states by introducing an auxiliary field. [12]

We also point out that the present results may be important to study the foundations of non-equilibrium statistical physics. For example, in the linear response theory of Kubo, [16] he assumed the unitary time evolution of a closed system. However, actually, the system is continuously measured over a time period longer than $1 / \omega$ when one measures, say, the AC conductivity at frequency $\omega$. Therefore, it is necessary for the validity of the linear response theory that the non-equilibrium state under consideration is stable against measurements. Theorem 3 suggests that such states must have the cluster property. This observation may become a foundation not only of non-equilibrium statistical physics but of non-equilibrium field theory, which is not established yet.

\footnotetext{
${ }^{7}$ For example, it is quite unlikely that a SB field for antiferromagnets could exist in laboratories.
} 


\section{ACKNOWLEDGMENTS}

The authors thank Prof. I. Ojima for discussions and suggestions.

* Present address: Department of Information Sciences, Tokyo University of Science, Chiba 278-8510, Japan.

[1] A. J. Leggett, Suppl. Prog. Theor. Phys. (Kyoto) 69, 80 (1980).

[2] W. H. Zurek, S. Habib and J. P. Paz, Phys. Rev. Lett. 70,1187 (1993), and references cited therein.

[3] More details will be presented in A. Shimizu and T. Miyadera, Phys. Rev. Lett., in press.

[4] R. Haag, Local Quantum Physics (Springer, Berlin, 1992).

[5] D. Ruelle, Statistical Mechanics: Rigorous Results (Benjamin, Reading, 1969).

[6] P. Horsh and W. von der Linden, Z. Phys. B72, 181 (1988).

[7] S. Miyashita, Qunatum simulations of condensed matter phenomena (eds. D. Dolland and J. E. Gabernatis, World Scientific, Singapore, 1990) p. 228.

[8] T. Koma and H. Tasaki, J. Stat. Phys. 76,745(1994)

[9] A. Shimizu and T. Miyadera, Phys. Rev. E 64, 056121 (2001).

[10] A. Shimizu and T. Miyadera, J. Phys. Soc. Jpn. 71 (2002) 56.

[11] A. Shimizu and T. Miyadera, Phys. Rev. Lett. 85, 688 (2000)

[12] H. Umezawa, H. Matsumoto and M. Tachiki, Thermo Field Dynamics and Condensed States (North Holland, Amsterdam, 1982).

[13] A. Shimizu, talk presented at The 4th Symposium on Quantum Effects and Related Physical Phenomena (December 20-21, 2000, Tokyo, Japan).

[14] A. Ukena and A. Shimizu, in preparation.

[15] This point was first suggested by G. M. Palma, K.-A. Suominen and A. K. Ekert, Proc. Roy. Soc. Lond. A (1996) 452 , 567.

[16] R. Kubo, J. Phys. Soc. Jpn. 12, 570 (1957). 